\title{
Enfrentándome a mí misma: réplicas a Natalia Scavuzzo, Alejandro Guevara Arroyo y Ezequiel Monti
}

\author{
Facing Myself: Answers to Natalia Scavuzzo, \\ Alejandro Guevara Arroyo and Ezequiel Monti
}

Verónica Rodríguez-Blanco*

Recepción: 20/02/2021

Evaluación: 25/02/2021

Aceptación final: 25/02/2021

\begin{abstract}
Resumen: en este ensayo, respondo a una serie de comentarios y críticas a mi artículo "Rastreando las críticas de Finnis al punto de vista interno de Hart: inestabilidad y el 'sentido y propósito' de la acción humana en el derecho" formulados por Natalia Scavuzzo, Ezequiel Monti y Alejandro Guevara Arroyo. Los autores discuten y formulan preguntas importantes en relación a la naturaleza y carácter de la acción humana y la metodología de la filosofía del derecho.

Palabras clave: teoría de la acción humana, Wittgenstein y la naturaleza del Derecho, metodología del caso central, H. L. A. Hart, John Finnis y Elizabeth Anscombe.
\end{abstract}

* Catedrática de Filosofía Política, Moral y Legal, Centro de Filosofía del Derecho, University of Surrey, Guild-ford, Reino Unido. Correo electrónico: v.rodriguez-blanco@surrey.ac.uk. El material de este trabajo se basa en mi artículo "Tracing Finnis's criticism of Hart's Internal Point of View: Instability and the 'Point' of Human Action in Law", en The Cambridge Companion to Legal Positivism, Cambridge University Press, forthcoming. Mi pro-fundo agradecimiento a Nelson Tepedino y Gabriela Scataglini por la cuidadosa traducción del presente texto. 


\begin{abstract}
I advance a number of responses to Natalia Scavuzzo's, Alejandro Guevara Arroyo's and Ezequiel Monti's comments and criticisms on my paper "Rastreando las críticas de Finnis al punto de vista interno de Hart: inestabilidad y el 'sentido y propósito' de la acción humana en el derecho". The authors raise important questions on the nature of human action and the methodology of legal philosophy.

Keywords: theory of human action, Wittgenstein and the nature of law, central case methodology, H.L.A Hart, John Finnis and Elizabeth Anscombe.
\end{abstract}

Es un placer haber leído las críticas tan sutiles e inteligentes de jóvenes investigadores, quienes prometen llevar la filosofía del derecho en Argentina y el mundo a una nueva era de altura intelectual. Me siento, pues, muy privilegiada de estar en este espacio y haber aprendido tanto. No hay mejor manera de honrar el pensamiento que imbuirse en una conversación crítica en busca de la verdad.

Trato de responder y reflexionar sobre los puntos y argumentos más importantes esgrimidos por Natalia Scavuzzo, Ezequiel Monti y Alejandro Guevara Arroyo, pero mis respuestas son deficientes en ocasiones, pues es claro que se abren puertas muy interesantes para indagar más profundamente sobre puntos álgidos y desacuerdos sustantivos.

\title{
1. Respuestas a las críticas formuladas por Natalia Scavuzzo
}

Scavuzzo hace una interesante exploración con relación a la conexión entre la teoría hartiana de la práctica social y la teoría wittgensteiniana de la filosofía del lenguaje. Como ella misma lo señala, no es del todo clara la influencia que tuvo Wittgenstein sobre Hart y esta es un área con una multiplicidad de interpretaciones e interesantes controversias. Hay una serie de contribuciones significativas con relación a esta coyuntura, pero, a mi parecer, un análisis justo y balanceado al respecto, iría más allá del ámbito de la presente discusión. 
En la segunda parte de su artículo, Scavuzzo analiza mi artículo "Peter Winch and HLA Hart: Two Concepts of the Internal Point of View" (Rodríguez-Blanco, 2007a), en el cual señalo, que debemos distinguir entre el punto de vista del participante, y el punto de vista práctico. Así, si hablamos de razones para la acción, pareciera que el punto de vista del participante no es lo suficientemente robusto como para establecer la conexión necesaria entre el agente y las razones para la acción. Como lo demuestro en mi libro Law and Authority Under the Guise of the Good (Rodríguez-Blanco, 2014 [2017]), las razones para la acción pertenecen al ámbito de la razón práctica y la teoría de la acción. Teorías adecuadas en ambos campos nos ayudan a esclarecer cómo las razones prácticas operan en la deliberación del agente y permiten la multiplicidad de acciones que ejecutamos en el ámbito del derecho. En el citado artículo (Rodríguez-Blanco, 2007a), señalo que Hart yerra en explicar el carácter de las razones para la acción en el ámbito jurídico. Sin embargo, Scavuzzo señala que identifico la falla de Hart como una falla en términos de una explicación de la obligatoriedad. Señala:

(es)... extraño sostener que "falla", es decir que tiene la intención de hacer cierta cosa o alcanzar cierto resultado pero no logra hacerlo. Los autores (Rodriguez-Blanco, Perry, Raz, Dworkin) parecen asumir una intención no expresada por Hart de resolver el conocido problema de si hay o no una obligación de obedecer el derecho (Scavuzzo, 2021, sección 5).

En mis obras, he dejado en claro, en numerosas ocasiones, que mi estudio sobre la razón práctica en el derecho no se centra en la importante pregunta sobre la obligatoriedad del derecho. Quizás, debo admitirlo, hay una cierta ambigüedad en mis primeros artículos. Sin embargo, en mi obra posterior, se deja claro que la pregunta por la normatividad del derecho debe conectarse a una explicación relacionada con las razones para la acción y la razón práctica, pero tales explicaciones no nos conducen a inferencias con respecto a la noción de la obligatoriedad del derecho. ${ }^{1}$

1 Recientes contribuciones sobre la obligatoriedad del derecho, incluye la obra de Stefano Bertea (Bertea, 2019). Nótese que mi rechazo a la idea de inferir un concepto de obligatoriedad a partir de la teoría de la acción de Anscombe y de la razón práctica tiene su raíz 
Comparto con Scavuzzo la idea de que la práctica social es fundamental para comprender el punto de vista interno, o más bien, lo que yo llamo el punto de vista de quien delibera o el de la primera persona. Scavuzzo nos reitera que el contexto de la práctica es esencial para comprender el punto de vista interno. Estoy de acuerdo con ella, pero también necesitamos precisar y analizar lo que entendemos por práctica social y por punto de vista interno.

A mi juicio, debemos resistir la tendencia de pensar que cuando presento mi análisis de la primera persona o de quien delibera, y del punto de vista práctico, y el fin de la acción como "características de lo bueno", $v$. $g$. lo valioso, lo que vale la pena, estamos refiriéndonos a algo que puede fácilmente representarse y articularse claramente. Es decir, que tenemos ideas preconcebidas y claras sobre lo bueno y valioso, que las mismas son objetivas. Por el contrario, aprendemos de ellas en los juegos de lenguaje y las formas de vida imbuidas de valor, de algo que "vale la pena". Esta característica institucional y social es explicada con gran riqueza en el artículo "On Brute Facts" escrito por Elizabeth Anscombe (Anscombe, 1958b) y el cual Zambrano y yo utilizamos para mostrar cómo lo inteligible, lo bueno, lo que vale la pena está inmerso en nuestras prácticas sociales (Rodriguez-Blanco, 2018). Sin embargo, el hecho de que lo inteligible, lo bueno, lo que vale la pena esté inmerso en nuestras prácticas sociales no lo hace una mera convención o un mero acuerdo. Más aún, tampoco lo hace un hábito, una simple repetición de acciones, una práctica sin reflexión o pensamiento. ${ }^{2}$ También creo que hay que evitar la idea de que cuando actua-

en la pregunta: ¿cómo concebir la noción de obligatoriedad bajo teorías de inspiración Aristotélica como la de Anscombe? La pregunta es importante, pero es un territorio delicado que requiere de un análisis cuidadoso y detallado. Véase la obra de Bernard Williams (Williams, 1993), quien se esfuerza en distinguir la moralidad kantiana con un fuerte énfasis en la noción de obligación y la ética Aristotélica, con énfasis en lo 'bueno' y valorativo. Véase también Anscombe (1958a).

2 En el pensamiento de Cavell (1979) se ve claramente este punto de vista. Cavell nos dice que el gran mensaje de la obra Investigaciones filosóficas (Wittgenstein, 1953 [2009]) es que debemos evitar la idea de que detrás del uso de las palabras que denotan lo mental como 'intención', 'creer', hay un evento privado que solo el hablante puede conocer y acceder. Esta es la fantasía del lenguaje privado. Pero el rechazo de esta fantasía, no nos lleva a decir que el significado de tal vocabulario mental es determinado por un acuerdo, o una convención 
Enfrentándome a mí misma: réplicas...

mos de conformidad con una característica de lo bueno, de lo valioso, de lo que vale la pena, esta caracterización sea de conformidad con un objetivo último y claro. También debemos resistir la idea de que estamos eligiendo, en el sentido volitivo por un lado, o reflexionando y sopesando por otro, previo a la elección. Me he afanado en evitar la idea de que poseer razones es un acto de sopesar. Por el contrario, poseer razones para la acción y, por lo tanto, considerar que algo 'vale la pena', o tiene una característica de lo bueno, puede originarse en la manera de interactuar social que aprendemos no solo como niños, sino constantemente. Pero ello no implica que sea un mero hábito y que no haya pensamiento que actúa como una comadrona en el proceso de deliberación de la acción práctica. La razón práctica no es un "know how" como se ha entendido con connotación habitual y sin representación. Verdaderamente, no hay una representación teórica, pero hay pensamiento sobre lo que deseamos, sobre lo bueno, lo valorativo.

Así, voy a intentar una nueva estrategia para clarificar lo que quiero expresar. Aristóteles usa la analogía del arte u oficios manuales o prácticos a fin de explicar la deliberación práctica, y su relación con el deseo, y lo valorativo. Imaginemos, pues una carpintera "Aurora", quien desea hacer una mesa. Las técnicas de carpintería las aprendió en su infancia, con su madre, y después las perfeccionó haciendo muchas mesas con su propia manera de entender la decoración en madera, las diferentes formas de mesa, los detalles y acabados finales. En una ocasión específica, Aurora piensa qué mesa ha de hacer. El cliente ha expresado su preferencia por una mesa redonda y grande para usar con amigos, pero también versátil para poder trabajar. Aurora piensa sobre el material adecuado, para hacer la mesa cómoda, no costosa y versátil. Diseña la mesa con unos detalles apropiados, compra y elige la madera perfecta, comienza a trabajar y piensa cómo refinar su técnica para hacer una mesa de conformidad con lo deseado y exigido. Si seguimos la estructura de la razón práctica, no diríamos que en cada movimiento físico de su acción, Aurora piensa en las características de lo

o el mero uso. Cierto, la riqueza del pensamiento que incorpora un vocabulario mental, se apoya en significados compartidos y aprendidos, pero eso no quiere decir que no hay algo que podríamos llamar 'mi pensamiento' y mis maneras de comprenderme, y formas de tratar de que los otros me reconozcan, o me evadan. Son los juegos del lenguaje que Cavell llama "acknowledgement and avoidance". 
bueno, pero si imaginariamente alguien le preguntara, podríamos decir que ella podría articular por qué hace lo que hace. No es que la estructura de la razón práctica se revele a través de la reflexión y articulación, pero se afirma que ella sabe lo que hace y por qué. Esto sustituye la inteligibilidad y la finalidad de su acción de hacer una mesa. Podemos encontrar una característica de lo bueno que da inteligibilidad a las acciones de Aurora. Recordemos: esto es solo una analogía. Las acciones humanas intencionales confrontan múltiples situaciones de gran complejidad, pero la posición que deseamos ilustrar es que causamos nuestras propias acciones intencionales porque sabemos, de manera transparente, qué y por qué estamos haciendo lo que efectivamente estamos haciendo. No decimos que la acción de Aurora, vista desde la perspectiva de la tercera persona es una interpretación. Más bien decimos que posee una estructura que se nos es inteligible porque compartimos una forma de vida.

Vamos a retornar al Hart de Scavuzzo, quien asevera que para Hart hay una práctica intersubjetiva, pues hay una práctica común y pública. Sin embargo, cuando me refiero al modelo hartiano en términos de actos mentales, no rechazo la posibilidad de intersubjetividad. La intersubjetividad es el resultado de creencias comunes con relación a la aceptación de las normas/reglas. He demostrado en mi libro (Rodríguez-Blanco, 2014 [2017]), a través de un análisis textual de la obra de Hart, que las creencias son entendidas como estados mentales. ¿Cuál sería pues la interpretación alternativa? Scavuzzo parece indicarnos que la noción wittgensteiniana podría dar anclaje a una visión alternativa. Mi duda en emplear el andamiaje wittgensteiniano se fundamenta en la idea de que la noción hartiana de "aceptación" pierde toda fuerza. Aceptación à la Wittgenstein se revela en la acción, en el seguir la regla y en el estar inmerso en los juegos de lenguaje. Como Stephen Mulhall (2001) lo ha demostrado en su interpretación de la obra de Wittgenstein (1953 [2009]), en Wittgenstein no hay interpretación, ni estado intermediario. Pero el actuar mismo, bajo esta interpretación del modelo wittgensteiniano, es precisamente aceptación. ¿Por qué Hart, si este es el modelo adecuado, se refiere explícitamente a una “aceptación”? Más aún, ¿por qué la "aceptación” es necesaria solo para algunos, los oficiales, y no para todos los ciudadanos si es un juego de lenguaje compartido? Todo el aparato hartiano pierde sentido bajo este esquema. 
Más aún, si simplemente participamos de los juegos de acción y lenguaje, entonces no se puede hablar de intersubjetividad, pues ello nos llevaría de nuevo al modelo de estados mentales. Ha de haber una subjetividad para encontrar intersubjetividad, diría yo, ¿o no?

\section{Respuestas a las críticas formuladas por Alejandro Guevara Arroyo}

Guevara Arroyo nos señala que "la metodología de la teoría del derecho trata sobre los procedimientos y criterios por medio de los cuales se logra que una cierta propuesta de concepto de derecho pueda ser considerada correcta o adecuada" (2021, sección 2). A partir de esto, Guevara Arroyo traza la subyacente concepción metodológica propuesta en mi artículo "Is Finnis Wrong?” (Rodríguez-Blanco, 2007). Así, en el mismo se explica el "caso central" o "foco central" como una metodología que intenta demarcar entre casos marginales del derecho y casos no-marginales o centrales. Correctamente, Guevara Arroyo señala que en mi obra el caso central es el punto de vista del agente práctico que delibera, quien ve el fin de la acción como algo valioso, que vale la pena, o algo bueno. En otras palabras, lo ve bajo la "apariencia del bien". Así, apelo a la filosofía de la acción de Anscombe (1957 [2001]) para explicar este punto de vista central o focal. Guevara Arroyo elabora una reconstrucción meta-teórica sobre el concepto de derecho propuesto en mi obra. El punto de partida de Guevara Arroyo es que hay dos elementos en todo concepto, los cuales son intensión y extensión. La primera se define por un conjunto de propiedades y relaciones, la segunda por aquello que cae bajo el concepto. Guevara Arroyo también nos dice que la corrección o validez de un concepto científico clasificatorio puede ser analizado desde la perspectiva de dos aspectos centrales: el problema del realismo de las propiedades, y el de la arbitrariedad de la clasificación. El primer problema se relaciona con las propiedades señaladas en la intensión del concepto las cuales están presentes en los individuos que pertenecen al conjunto de la extensión. El aspecto crucial que Guevara Arroyo desea establecer se ejemplifica en el siguiente párrafo: 
para sustentar su corrección, validez o adecuación material, los conceptos necesariamente han de apelar a teorías e hipótesis que no son ni conceptos clasificatorios ni sistemas clasificatorios. Las relaciones metodológicas que se presentan entre las teorías e hipótesis explicativas y los mentados conceptos son variadas, pero aquí lo que quiero dejar sentado es que la corrección material de los conceptos clasificatorios parece parasitaria del estatus epistémico de teorías y modelos explicativas que son mucho más que aquellas (Guevara Arroyo, 2021, sección 4.2)

A partir de este marco, Guevara Arroyo formula dos preguntas centrales, (a) ¿Es que acaso hay una analogía entre el concepto de derecho y los conceptos científicos clasificatorios entendidos en su específica relación con las teorías explicativas? (b) ¿Cómo mi metodología puede justificar la adecuación material de un cierto concepto de derecho? El autor ve la justificación epistémica como fundamental. Acertadamente, Guevara Arroyo señala que los participantes de la práctica no siempre comparten todas las propiedades de la intensión del concepto (Rodríguez-Blanco, 2007). Sin embargo, asevera que estos conceptos son efectivamente conceptos clasificatorios; no obstante, el problema genuino emana con la corrección material del concepto.

Más aún, Guevara Arroyo sugiere los siguientes comentarios críticos. Para él, existe una tensión entre mi artículo "A Defence of Hart's Semantics as Non-Ambitious Conceptual Analysis" (Rodríguez-Blanco, 2003) y mi obra posterior. En ese artículo interpreto que el método utilizado por Hart es el de análisis conceptual no ambicioso. Me apoyo en la concepción de Jackson con relación a una aproximación al análisis conceptual, la cual se vislumbra como más fructífera. Así, el teórico puede desarrollar un concepto basado en las intuiciones y teorías "folk" de los hablantes, sin embargo, esta es una aproximación modesta, pues solo esculpe un aspecto metafísico del concepto. El análisis conceptual encuentra un límite y consideraciones empíricas pueden jugar un rol importante a fin de revisar nuestro análisis conceptual inicial. De conformidad con Guevara Arroyo, hay una tensión entre este artículo y su posición con respecto al análisis conceptual no ambicioso y mi obra posterior. Sin embargo, en el artículo mencionado solo reconstruyo la metodología de Hart y sugiero una interpretación que considero más fructífera y con mayor poder explicativo. Mi 
obra posterior, especialmente mi libro (Rodríguez-Blanco, 2014 [2017]), intenta avanzar una teoría de la normatividad y autoridad jurídicas, y la metodología del caso central se asume como implícita, pero se elabora de manera más sofisticada y detallada que en la obra de Finnis, pues hay un desarrollo completo de la noción de razón práctica que corre paralela a las ideas de intención y razones para la acción.

Guevara Arroyo insiste en el problema de la corrección del concepto, que a mi parecer es una pregunta importante. Sin embargo, la manera en que se formula su solución me parece problemática. La solución de Guevara Arroyo sería la siguiente:

... se formula una potente teoría explicativa, llamémosle $\varepsilon$, que establece sólidas leyes sociales sobre el funcionamiento del Derecho en buena parte del mundo. Esta utiliza un concepto $\gamma$ de Derecho, bastante distinto $\alpha_{1}$. Como si fuera poco, la existencia de las propiedades señaladas en la intensión de $\gamma$ puede ser explicada a partir de potentes programas científicos $\delta$, conectándolas con propiedades más profundas del mundo ( $v$.g. mecanismos neuro-psicológicos presentes en los humanos) (Guevara Arroyo, 2021, sección 5).

Se llama $\alpha_{1}$ el concepto propuesto por mi metodología, es decir, la metodología del caso central o focal, el cual es el caso en el que el agente se imbuye en la deliberación práctica. Los otros casos son identificados con relación a este caso central o focal a manera de "semejanza" (Rodríguez-Blanco, 2007). Para Guevara Arroyo, no podemos justificar la aseveración de que el nuevo concepto de derecho $\gamma$ no ha superado los problemas y limitaciones del concepto propuesto por mi metodología, $\alpha_{1}$ El nuevo concepto $\gamma$ no es arbitrario y sus propiedades existen realmente. Así, nos reitera Guevara Arroyo, no tenemos ninguna ventaja al insistir que $\alpha_{1}$ resulta en conformidad con la naturaleza del derecho. ¿Por qué no decir que tal teoría $\varepsilon$ ha revelado el verdadero concepto de derecho? ¿Por qué no es una respuesta sólida a qué es el derecho? Guevara Arroyo acepta que la metodología finniana (Finnis, 1980) y mi propia propuesta (Rodríguez-Blanco, 2007) revelan cosas interesantes del mundo social, pero asevera que no es solo a través de este concepto que se da respuesta al problema de la naturaleza del derecho. 
Creo que hay una equivocación con la formulación de Guevara Arroyo con respecto al proyecto trazado tanto por mí, como por Finnis. La propuesta es la siguiente. El método del caso central no es el único método y concepto para desentrañar la naturaleza del derecho. He argumentado en mi libro (Rodríguez-Blanco, 2014 [2017]) que el punto de vista de la razón práctica provee una prioridad conceptual y lógica, a partir de la cual cualquier otra explicación del derecho ha de depender. Me he referido a esta posición en términos de una concepción "naïve", en oposición a una posición teórica, epistémica, ontológica o científica. Si hay explicaciones científicas no reductivistas y exitosas, las mismas han de explicar nuestras prácticas, las cuales he tratado de demostrar son inevitablemente valorativas. En otras palabras, la compleja fenomenología de lo que hacemos en las prácticas normativas características del derecho, que van desde encarcelar a presos, proteger derechos humanos, reformular conceptos de libertad, permitir y proteger nuevas prácticas sexuales, permitir y proteger nuevas prácticas de posesión de nuestro cuerpo, por mencionar a unas pocas, son la fenomenología esencial que debemos comprender, para poder continuar haciéndolas, mejorándolas, comprendiéndolas, explicándolas. Por supuesto, si la explicación es científica y reductivista, entonces nuestras concepciones más preciadas, por ejemplo la de que el agente ' $\mathrm{x}$ ' es responsable por el asesinato de tal persona $\mathrm{y}$ ha de ser castigado, pierden todo sentido. Esto no quiere decir que no hay espacio para nuevos descubrimientos neurocientíficos que nos fuercen a re-evaluar nuestras prácticas y lo que consideramos valioso y bueno. Sin embargo, como lo han demostrado filósofos en el área (Pardo, 2015), estos descubrimientos dependen de cómo demarcamos las prácticas. Hay espacio para una visión no reductivista, pero lo único que he tratado de argumentar es que no podemos perder de vista la fenomenología primaria de cómo vivimos nuestras prácticas sociales. En el nuevo mundo teórico de Guevara Arroyo queda la duda si la explicación es identificable como una explicación de la acción humana. 


\section{Respuestas a las críticas formuladas por Ezequiel Monti}

Monti comienza su reflexión con una pregunta clarificadora que me ayuda a esclarecer mi motivación y el argumento central esgrimido en mi artículo "Rastreando las críticas de Finnis al punto de vista interno de Hart: inestabilidad y el 'sentido y propósito' de la acción humana en el derecho" (Rodríguez-Blanco, 2021). Monti expresa sus dudas de que mi crítica a Hart esté conectada con la crítica formulada por Finnis en el capítulo primero de su libro Natural Law and Natural Rights (Finnis, 1980). Para Monti, la crítica de Finnis a Hart se limita a establecer la idea de que la teoría de Hart, y específicamente el llamado "punto de vista interno", no puede demarcar un límite al tipo de punto de vista interno, al cual el participante de la práctica jurídica toma como central. Así, una vez que aceptamos que el participante de la práctica jurídica asume el punto de vista interno, pareciera, de conformidad con Finnis, arbitrario señalar que el punto de vista interno no puede ser moral. En otras palabras, la crítica de Finnis se limitaría a decir: "al decir que hay un punto de vista interno, admitimos que puede haber una multiplicidad de puntos de vista internos, y por supuesto el más importante de todos, sería el punto de vista ético". La siguiente metáfora nos ilumina el punto: si usted deja salir al genio de la botella, no puede controlar su rol. En este caso el genio de la botella sería el "punto de vista interno". Entiendo que la crítica de Finnis se centra en la vaguedad y la necesidad de aclarar el "punto de vista interno". Es un punto iluminador, sin embargo, el mismo Finnis, no desarrolla detalladamente y propiamente una teoría ${ }^{3}$ que nos permitiría entender qué es el punto de vista interno y por qué ha de expandirse a un punto de vista ético. Asumir que la crítica es simplemente un problema de hasta dónde podemos extender lo "interno" nos llevaría a argumentos petitio principi. Así, los hartianos afirmarían que el punto de vista interno adquiere forma y significación dentro de la práctica jurídica misma. Los teóricos no-hartianos, como Finnis, afirmarían que se trata de incorporar la dimensión evaluativa y de cómo la incorporamos (Dickson,

3 En una serie de importantes ensayos escritos a los largo de su carrera académica explora qué es la intención y la noción la aplica a problemas álgidos como la teoría de la responsabilidad extracontractual (véase especialmente la parte tercera de sus obra editada [Finnis, 2011]). 
2001). En el caso de los primeros, se está asumiendo que el derecho es fundamentalmente una práctica social, en el caso de los segundos, se está dado por sentado que el derecho ha de incorporar una perspectiva ética. A mi juicio, esta simplificación del argumento no nos llevaría a ningún progreso teórico. En contraste, veo mi contribución como una expansión de la crítica de Finnis a fin de evitar argumentos petitio principi, es decir, caer en la falacia de tomar como punto de partida precisamente la premisa que queremos demostrar. En Rodríguez-Blanco (2021) deseo mostrar que debemos recurrir a la teoría de la acción a fin de elucidar la manera como actuamos y como seguimos las normas jurídicas, y de esta forma elucidar qué exactamente debemos entender por el llamado "punto de vista interno". La idea es que reflexiones y escudriñamientos sobre las teorías de la acción, nos hagan explícito lo que está implícito en la obra hartiana y la crítica de Finnis. Mi esperanza es que al final de la reflexión, el lector haya adquirido claridad sobre por qué el "punto de vista interno" no puede dar estabilidad y por lo tanto el participante de la práctica jurídica, armado con el punto de vista interno hartiano, no puede distinguir entre sistema jurídicos benevolentes y malvados, algo que todos nosotros podemos hacer ordinariamente y sin ningún esfuerzo.

Así, asumo que la defensa de una teoría de la acción adecuada nos puede mostrar que cuando actuamos siguiendo una norma jurídica, cuya estructura es sostenida por razones para la acción, estamos actuando de conformidad con una descripción de lo bueno o valorativo, visto desde el punto de vista del agente que realiza la acción. Esta perspectiva es primaria y primitiva, de conformidad con la fenomenología y lenguaje de nuestras acciones intencionales. De conformidad con Monti, mi argumento se puede reducir a lo siguiente: "El punto de vista hartiano sería inestable porque solo accidentalmente puede conducir a comprender el propósito o sentido de las acciones en el contexto jurídico. Esto, a primera vista, no tiene mucho que ver con la inestabilidad que denuncia Finnis" (Monti, 2021, sección 2). Si el "punto de vista interno" à la Hart es una aceptación formulada como un conjunto de creencias concebidas como estados mentales, entonces se podría concluir que la dimensión valorativa, $v . g$. lo bueno, lo correcto, lo valioso, lo malévolo, lo pernicioso, en tales creencias no juegan ningún rol en la determinación de la regla a seguir y sus razones 
Enfrentándome a mí misma: réplicas...

subyacentes. Es por eso que Finnis afirma que el participante hartiano no puede distinguir un régimen benevolente de un régimen malévolo, pues para Hart, señalo en mi artículo (Rodríguez-Blanco, 2021), la aceptación de la regla desde el punto de vista interno, es simplemente una creencia y por tanto, un estado mental que el teórico de la acción atribuye al participante. ${ }^{4}$ Por supuesto, afirmo en mis estudios sobre el tema, esta explicación teórica juega un rol importante, y Monti también lo reconoce, sin embargo no es una función central o primaria. Tampoco, a mi juicio, es "complementaria", como Monti asevera. Mi argumento es que la explicación de la acción en términos de la primera persona o la perspectiva deliberativa es primaria o central. Quiero decir, las razones para la acción, para el agente que delibera, están concatenadas con otras razones, y al final de este proceso deliberativo, el agente encuentra su acción inteligible debido a que ve el fin de la acción como algo "bueno" o "valioso". Así, lo valorativo está en el centro de la acción y juega un rol central. Este fin disfrazado como algo "bueno" que ve el actor da inteligibilidad a toda la acción. Así, por ejemplo, el agente al parar su vehículo frente al semáforo rojo, presiona el freno a fin de parar el vehículo, y para el vehículo a fin de obedecer la señal de tránsito, y obedece la señal de tránsito a fin de no chocar con otros vehículos

4 Es interesante que Monti utiliza una combinación de la metodología anscombiana de la pregunta "por qué" para elucidar la estructura de la acción intencional. Pero, por otro lado, confusamente, acepta la teoría davidsoniana de la acción, en términos de creencias vistas como estados mentales. Nótese que para Davidson (Davidson, 1963) la manera de elucidar la acción intencional es a través de la interpretación caritativa. Así, asumimos que los actores son racionales y les atribuimos deseos y creencias como razones primarias para la acción. En otras palabras, para Davidson, es desde el punto de vista teórico o de la tercera persona que atribuimos al agente la descripción intencional de la acción.

Lamentablemente, Monti no aclara por qué se desvía de la manera davidsoniana de entender y atribuir estados mentales. A mi juicio, aceptar la metodología anscombiana de "por qué", nos lleva a un territorio distinto del de la explicación teórica, pues nos adherimos a la explicación del agente que delibera. También he de aclarar que su propuesta de una teoría de los estados mentales que no es causal me parece interesante, pero no desarrollada, y por razones comprensibles debido al espacio limitado de la presente discusión. Si no es causal, entonces ¿cómo los actos mentales producen el movimiento de mi cuerpo? Podríamos decir que la conexión es racional, pero ahora nos estamos acercando a la teoría de la acción de Anscombe, por no mencionar a Kant. Más aún, ¿por qué insistiríamos en llamarlos estados mentales si la conexión es racional? Las ventajas empíricas de la teoría davidsoniana se perderían. 
o atropellar a los peatones que cruzan la calle. Este último fin "visto como bueno" ilumina y da sentido al resto de las acciones, es decir al presionar el freno, parar el vehículo, etc. ¿Por qué el rol de esta descripción es primaria y no complementaria?, se puede preguntar el lector. Lo cóncavo y lo convexo son complementarios, porque al combinar una figura cóncava con una figura convexa podemos observar que hay un enaltecimiento de las características de cada figura geométrica, la cóncava y la convexa. Por el contrario, he intentado demostrar, que la explicación de la acción en términos de la "apariencia del bien" es primaria y naïve con respecto a la explicación teórica, por lo siguiente. La explicación teórica debe rastrear la estructura de concatenación de las razones como son vistas y descritas por el agente, si tal explicación desea capturar la acción intencional del participante jurídico, quien sigue las normas jurídicas. La prueba del éxito de la explicación teórica es si refleja, o más bien es un espejo o representación certera de lo que agente hizo tal y como como es concebido por ella. La explicación teórica explica lo que el agente hizo, pero lo que el agente hizo es determinado por lo que ella deliberó y la manera como ella concibe la acción. Aclaremos este punto con el ejemplo dado por Monti. Así, Juan se aterroriza y sale de la habitación corriendo al ver una serpiente. Sus amigos se ríen, nos dice Monti, pues no es una serpiente real, es simplemente un juguete. Monti nos dice que durante la cena Juan explicó que confundió la serpiente de juguete con una serpiente real. Monti añade:

Ahora supongamos que queremos explicar por qué Juan salió corriendo sin comprometernos con la existencia de la serpiente. Entonces, nuevamente, no nos queda otra opción que explicar su acción en virtud de sus creencias y actitudes antes que en virtud de como él mismo hubiera explicado su acción en el momento en que estaba realizándola, esto es, en términos de serpientes en lugar de creencias acerca de serpientes. Esto podría explicar por qué Hart, cuya pretensión consistía en construir una teoría del derecho que haga justicia tanto al punto de vista externo como al interno, se limita a explicar las acciones en el contexto jurídico en términos de estados mentales (Monti, 2021, sección 3.4) 
Pero ¿por qué hemos de explicar la acción de Juan sin comprometernos con la existencia de la serpiente? Juan diría: "salí corriendo porque había una serpiente, las serpientes son venenosas y les tengo miedo". En su deliberación, es valioso preservar la vida, evitar cosas peligrosas. Por supuesto, después de salir corriendo y al ver que era un juguete, puede decir: "Ah, me equivoqué en mi acción, pues la serpiente era realmente un juguete". No es cierto que su intención es equivocada, al actuar intencionalmente trataba de evitar la mordedura de la serpiente. Su error no es en la intención, sino en las circunstancias de la acción. Pero no necesitamos el marco de la teoría de los estados mentales para comprender la inteligibilidad de la acción de Juan y su error. Si siempre racionalizáramos las acciones en términos de estados mentales y las capturáramos como racionalizaciones, no habría espacio para el error, el perdón, la culpa, el arrepentimiento. Precisamente, la existencia de la serpiente, apreciada como real y peligrosa, nos permite decir que la acción fue intencional. La premura de Juan en salir del cuarto no fue algo meramente accidental y el modelo de la "apariencia del bien" nos hace tal acción inteligible y los errores palpables a efectos de la responsabilidad y culpabilidad. Si Juan destroza la serpiente-juguete podemos comprender por qué lo hizo y podemos excusarlo o justificarlo.

Pero imaginemos un escenario alternativo. Juan sabía que la serpiente era un juguete y sale corriendo, durante la cena nos cuenta que lo hizo para que sus amigos se rieran. Pero imaginemos que como filósofos le atribuimos una racionalización entre el tiempo de la acción y antes de cenar, cuando la información sobre la intención de Juan se nos hace palpable. Explicamos la acción a través de la atribución de la creencia de estar en presencia de una serpiente y el deseo de evitar ser mordido por la serpiente. Sin embargo, sabemos que esta no era la intención de Juan. ¡Su intención era gastarnos una broma! Así, el modelo de atribución de estados mentales falla si ignora el punto de vista del agente o de quien delibera.

En algún punto, Monti argumenta que la tesis de la acción en términos de la apariencia del bien es primaria, pero nos dice, que la explicación en términos de estados mentales compite con la misma, pues al utilizar la explicación en términos de creencias, no perdemos nada en la traducción (Monti, 2021, sección 3.5). En su ejemplo, Juan pagó el alquiler porque es bueno cumplir con las promesas, pero también podemos decir Juan pagó la 
renta porque "cree que debe cumplir sus promesas y cree que ha hecho una promesa”. Sin embargo, argumento, precisamente, la explicación última es inteligible porque depende de la primera, es decir, sabemos que Juan pagó el alquiler para cumplir su promesa y cumplir promesas es algo bueno para Juan. Este punto confirma la primacía del modelo de la "apariencia del bien". Debemos señalar, que este no es explicativo como el modelo mental o teórico, pero más bien es naïve o primitivo porque se centra en la inteligibilidad desde el punto de vista del agente que delibera. Es primitivo en el sentido básico de cómo experimentamos nuestras acciones en el mundo.

Supongamos el siguiente ejemplo. No sabemos nada sobre Juan. En la noche temprano, estamos sentados frente al edificio donde vivimos, Juan es nuestro vecino y vemos su figura en la noche. Observamos que coloca un cheque por debajo de la puerta de la encargada del edificio, quien colecta las rentas cada mes. Precisamente, es el día del pago mensual de la renta por parte de todos los inquilinos. Davidson nos dice que a fin de entender la acción debemos presumir la racionalidad caritativa y atribuir al agente creencias y deseos que van a explicar la acción. Así, inferimos que Juan paga la renta porque "cree que ha prometido pagar la renta cada mes", y "desea cumplir con sus promesas". Sin embargo, Juan sufre de sonambulismo y esa noche al entregar el cheque lo hizo como sonámbulo. El resultado es que la acción atribuida como estado mental no corresponde a la acción intencional. El argumento es que solo al indagar sobre su acción desde el punto de la primera persona como agente que delibera podemos hacer inteligible la acción de Juan y entender que no actuó intencionalmente, más bien estaba en estado de sonambulismo. El modelo anscombiano nos permite delimitar acciones intencionales y no intencionales, pero también nos permite darle inteligibilidad a la acción, v. g., nos es inteligible que Juan paga la renta y cumple su promesa porque él lo ve como algo valioso o bueno.

Finalmente, he de señalar, que en ninguna parte asevero que de lo bueno se sigue lo obligatorio. A Monti le preocupa lo siguiente con respecto a lo valorativo y lo obligatorio:

Ella sostiene, correctamente, que para que una persona guíe su conducta en virtud de reglas jurídicas debe considerarse que es bueno o valioso actuar conforme a ellas. Sugiere además que ello 
Enfrentándome a mí misma: réplicas...

implica que las reglas son obligatorias, en la medida en que lo son, porque es bueno o valioso actuar como requieren. Esto, empero, no se sigue. Es posible, en efecto, que las reglas sean obligatorias en virtud de otras consideraciones, que no tienen nada que ver con que sea bueno o valioso realizar la acción requerida (Monti, 2021, sección 5).

Sin embargo, he de aclarar que ni en mi libro, ni en mi artículo objeto de discusión, asumo que de lo valioso se infiere lo obligatorio. Más aún en un symposium publicado sobre mi libro, donde autores comentaron sus argumentos, Webber se pregunta si a partir de mis argumentos se puede inferir la obligatoriedad. En mis réplicas rechacé de manera categórica la conexión. ${ }^{5}$

Considero que la pregunta sobre lo obligatorio en el ámbito del derecho es central y muy importante, pero no creo que una teoría de la acción con relación a 'la apariencia' del bien sea lo suficientemente iluminadora al respecto.

Creo que Monti y yo estamos de acuerdo en un número sustantivo e importante de puntos y argumentos. No obstante, he querido enfatizar las diferencias con la esperanza de continuar la discusión entre ambos, pero también para invitar a otros interlocutores a continuar indagando en estos temas.

5 En el symposium de mi libro Law and Authority Under the Guise of the Good, cuyo título es: "The Why-Question Methodology, The Guise of the Good and Legal Normativity" (Rodríguez-Blanco, 2017), le respondo a Webber de la siguiente manera: "Law and Authority does not aim to show the way law is connected to the common good generating the obligatoriness of law. This has masterfully been done by John Finnis's Natural Law and Natural Rights. The aim of the book was to focus attention on the structure of practical reason and to engage in an analysis of how the law intervenes and participates in the practical reasoning and action of the addressees of the law when it already does so. I argue that an explanation of how this happens is an explanation of the normativity of law. The normativity of law is an explanation of how we engage and use reasons for actions and I try to show that reasons for actions are necessarily connected to values and are the grounding of legal directive and legal rules. I do not engage in an explanation of legal obligation and the word 'obligation' does not appear in the entire text, apart from some occasions, where I discuss what other authors have said about it, e.g. Austin, Hart, Raz". 


\section{Bibliografía}

Anscombe, E. (1957 [2001]). Intention. Oxford: Blackwell.

Anscombe, E. (1958a). Modern Moral Philosophy. Philosophy, 33(124), 1-19.

Anscombe, E. (1958b). On Brute Facts. Analysis, 18(3), 69-72.

Bertea, S. (2019). A Theory of Legal Obligation. Cambridge: Cambridge University Press.

Cavell, S. (1979). The Claim of Reason: Wittgenstein, Scepticism, Morality and Tragedy. Oxford: Oxford University Press.

Davidson, D. (1963). Actions, Reasons and Causes. The Journal of Philosophy, 60(7), 685-700.

Davidson, D. (1973). Radical Interpretation. Dialectica, 27, 314-28.

Dickson, J. (2001). Evaluation and Legal Theory. Oxford: Hart.

Finnis. (1980). Natural Law and Natural Rights. Oxford: Oxford University Press.

Finnis, J. (1980). Natural Law and Natural Rights. Oxford: Clarendon Press. Finnis, J. (2011). Intention and Identity. Oxford: Oxford University Press.

Guevara Arroyo, A. (2021). Naturaleza y concepto de Derecho a los ojos de Rodríguez-Blanco: un análisis naturalista de su propuesta metodológica. Discusiones, 26(1).

Monti, E. (2021). Rodríguez-Blanco sobre el carácter inestable del punto de vista interno. Discusiones, 26(1).

Mulhall, S. (2001). Inheritance and Originality. Oxford: Oxford University Press.

Pardo, M. S. (2015). Minds, Brains and, Law: The Conceptual Foundations of Law and Neuroscience. Oxford: Oxford University Press.

Rodríguez-Blanco. (2003). A Defence of Hart's Semantics as NonAmbitious Conceptual Analysis. Legal Theory, 9(2), 99-124.

Rodríguez-Blanco. (2007). Is Finnis Wrong? Legal Theory, 13, 257-283.

Rodríguez-Blanco, V. (2007a). Peter Winch and H.L.A Hart: Two Concepts of the Internal Point of View. Canadian Journal of Law and Jurisprudence, 20(2), 453-473.

Rodríguez-Blanco, V. (2014 [2017]). Law and Authority Unde the Guise of the Good. Oxford: Hart/Bloomsbury Publisher.

Rodríguez-Blanco, V. (2017). “The Why-Question Methodology, The Guise of the Good and Legal Normativity”. Jurisprudence, 8(1), 127-142. 
Rodríguez-Blanco, V. (2021). Rastreando las críticas de Finnis al punto de vista interno de Hart: inestabilidad y el 'sentido y propósito' de la acción humana en el derecho. Discusiones, 26(1).

Rodriguez-Blanco, V. y. (2018). One Myth of the Natural Law Theory: Reflecting on the 'Thin' View of Legal Positivism. Ratio Juris, 31(1), 9-32.

Scavuzzo, N. (2021). Hart y el "sentido y propósito" del punto de vista inteno. Discusiones, 26(1).

Williams, B. (1993). Ethics and the Limits of Philosophy. London: Fontana Press.

Wittgenstein, L. (1953 [2009]). Philosophical Investigations (4th Edition ed.). Oxford: Blackwell. 